\title{
Fecundidad y esfuerzo reproductivo de Petrolisthes granulosus (Guérin, 1835) en Iquique, Chile (Decapoda, Anomura, Porcellanidae)
}

\author{
Laura López ${ }^{1}$, Teresa Jeri ${ }^{2}$, Claudio González ${ }^{3}$ y Sergio Rodríguez ${ }^{4}$ \\ ${ }^{1}$ Facultad Ciencias Exactas y Naturales (UBA), Fisiología Animal Comparada \\ Ciudad Universitaria Pabellón 2, 1428, Buenos Aires, Argentina \\ ${ }^{2}$ Alfred Wegener Institut, Bremerhaven, Alemania \\ ${ }^{3}$ Departamento Ciencias del Mar, Universidad Arturo Prat, Iquique, Chile \\ ${ }^{4}$ Facultad Ciencias Exactas y Naturales (UBA), Casilla de Correo 94, Sucursal 37(B) \\ 1437 Buenos Aires, Argentina
}

\begin{abstract}
RESUMEN: Se estudió la fecundidad evaluada como número de huevos por hembra y el rendimiento reproductivo de Petrolisthes granulosus durante marzo de 1996 en Iquique, Chile. El número de huevos no mostró diferencias significativas entre estadíos embrionarios, aunque se detectó un incremento en el volumen medio por huevo del $45,8 \%$, atribuible al mayor contenido porcentual de agua y un consecuente decremento en el peso seco de los huevos $(24,3 \%)$. El número total de huevos mostró una fuerte dependencia con la talla de la hembra. El esfuerzo reproductivo total fue del $10 \%$ y entre estadíos disminuyó significativamente hacia fines del desarrollo embrionario.
\end{abstract}

Palabras claves: Petrolisthes granulosus, fecundidad, esfuerzo reproductivo.

\section{Fecundity and reproductive output of Petrolisthes granulosus (Guérin, 1835) in Iquique, Chile (Decapoda, Anomura, Porcellanidae)}

\begin{abstract}
Fecundity (number of eggs carried by a female) and reproductive output were studied in Petrolisthes granulosus during March 1996 in Iquique, Chile. There was no egg lost during embryonic development, but there was an increment in egg volume of $45.8 \%$ during the incubation period, which was due to an important increase in water content. The egg dry weight decreased in $24.3 \%$. The total egg number was related to female size. The reproductive output for all females was nearly $10 \%$ but there was a significative decrease at the end of embryonic development.
\end{abstract}

Key words: Petrolisthes granulosus, fecundity, reproductive output.

\section{INTRODUCCION}

La fecundidad ha sido tradicionalmente definida como el número de huevos puestos por hembra y ha permitido estudios comparativos entre poblaciones de la misma especie a nivel interespecífico o entre taxa superiores (Annala, 1991; Corey y Reid, 1991; Hines, 1982, 1988 y 1991; Ivanova y Vassilenko, 1987). Stechey y Somer (1995) amplían este concepto definiendo «potencial de fecundidad» (número de oocitos vitelogénicos en el ovario), «fecundidad realizada» (número de huevos puestos por hembra) y «fecundidad actual» (número de larvas eclosionadas). El concepto tradicional sería equivalente al de fecundidad realizada.

Los estudios entre la fecundidad de la hembra y su talla y peso son importantes para comprender los patrones subyacentes a la dinámica, productividad y reclutamiento de las poblaciones (Ivanova y Vassilenko, 1987; Reid y Corey, 1991).

Se ha observado que las relaciones entre medidas morfométricas (volumen del caparazón en carideos y área del abdomen en porcelánidos y braquiuros) representan mejor la relación que existe entre el tamaño de la hembra y la cantidad de huevos portados en el espacio físico disponible (Corey y Reid, 1991; Lardies y Wehrtmann, 1996). El mayor determinante para el tamaño de la puesta es el espacio disponible para la acumulación de vitelo en la cavidad corporal (Hines, 1982, 1988, 1991). 
La inversión reproductiva se puede calcular en un número variable de formas. El verdadero esfuerzo reproductivo es usualmente definido como la fracción de la energía total orientada a la reproducción. Dada la dificultad de medir ésto, la forma habitual de medir la inversión es el esfuerzo reproductivo (RO):

$$
\mathrm{RO}=\frac{\text { peso seco total de huevos }}{\text { peso seco de la hembra }}
$$

La familia Porcellanidae está representada por 16 especies en Chile, 13 de las cuales se encuentran en la I Región (Haig, 1960).

En la zona de Huaiquique, Iquique, la especie $P$. granulosus se distribuye en el intermareal rocoso bajo bloques de gran tamaño, presentando una distribución agrupada independiente de sexo y ta1la. La fauna acompañante observada está representada por ejemplares de equinodermos, gasterópodos, actinias, otros porcelánidos como $P$. violaceus y algunos grápsidos . Similar observación ha sido realizada por Sanhueza et al. (1975) para El Tabo, ubicado al sur de Valparaíso.

El presente trabajo tiene por objetivo aportar mayor información sobre $P$. granulosus en lo referente a su fecundidad y esfuerzo reproductivo en la zona costera de Iquique, Chile. A través de este documento se compararan los resultados obtenidos con otras especies del género Petrolisthes de la costa del Pacífico.

\section{MATERIALES Y METODOS}

Durante marzo de 1996 se recolectaron 79 hembras ovígeras de Petrolisthes granulosus en un rango de talla comprendido entre 4,3 y 8,0 mm de longitud de caparazón en el intermareal rocoso de playa Huaiquique, Iquique (20¹9'S, $\left.70^{\circ} 08^{\prime} \mathrm{W}\right)$. Los ejemplares fueron transportados vivos al laboratorio y colocados en recipientes plásticos individuales de $50 \mathrm{ml}$ de capacidad, en un volumen de $15 \mathrm{ml}$ de agua de mar previamente filtrada con un tamiz de $180 \mu$. A cada una de las hembras ovígeras se le midió el largo máximo del caparazón (LC), con un medidor tipo vernier (precisión 0,05 mm). Posteriormente, se extrajo la masa ovígera y se contó la totalidad de los huevos por hembra. El desarrollo embrionario de los huevos fue clasificado en tres estadíos, utilizando los criterios indicados por Wehrtmann (1990).
Además, se determinó el color de los huevos para establecer una escala macroscópica de desarrollo embrionario y relacionarla con la previamente mencionada. De esta manera se definieron tres colores: morado (M), morado rojizo (MR) y morado anaranjado (MA). A cada hembra se le midió el diámetro mayor y menor de 10 huevos bajo microscopio estereométrico (Leitz Laborlux K). A partir de estos datos se determinó el volumen por huevo mediante la fórmula para huevos esféricos (Turner y Lawrence, 1979).

Posteriormente se determinó el volumen de la masa ovígera para cada hembra como el volumen promedio por huevo por el número de huevos por hembra. Luego se llevaron a estufa durante 48 horas a $70^{\circ} \mathrm{C}$ para determinar el peso seco de la masa total de huevos (PTSH) y el peso seco de la hembra (PSF) con una balanza Sartorius de precisión 0,1 $\mathrm{mg}$. Con estos datos se determinó el esfuerzo reproductivo.

La relación entre LC ( $\mathrm{mm}$ ) y el número de huevos por hembra fue analizada mediante regresión lineal. La significación de los resultados fue probada estadísticamente mediante el test de Student $(\mathrm{p}>0,05)$.

\section{RESULTADOS Y DISCUSION}

La Tabla 1 muestra la relación entre el largo máximo de caparazón y el peso seco de las hembras y el estadío de desarrollo embrionario de los huevos portados. En la Tabla 2 se indica el número de huevos (fecundidad), volumen por huevo, volumen de la masa de huevos y esfuerzo reproductivo para las tres categorías indicadas. En todos los casos resultó $\mathrm{p}<0,05$.

El grado de desarrollo embrionario y su relación con la coloración de la masa de huevos se indican en la Tabla 3. La relación entre la talla de la hembra (LC) y el número de huevos entre estadíos no presento diferencias significativas, siendo las hembras de mayor tamaño las que portaron mayor número de huevos (Fig. 1).

La talla mínima de las hembras ovígeras 40 fue de 4,3 mm de longitud cefalotoráxica, similar a los valores hallados por Antezana et al. (1965) y Sanhueza et al. (1975) de 5,0 y 4,6 mm respectivamente. Las diferencias observadas entre las localidades de Iquique, El Tabo y Valparaíso son leves, las que podrían indicar variaciones latitudinales. Relaciones entre los patrones reproductivos y la la- 
Tabla 1. Relación entre el largo máximo de caparazón (LC) y peso seco de las hembras (PSF) y el estadío de desarrollo embrionario de los huevos portados por las hembras.

Table 1. Relationship between the maximum carapace length (LC) and females dry weight (PSF) and the embrionic development stage of eggs carried by females.

\begin{tabular}{|ccccccccccc|}
\hline & \multicolumn{4}{c}{ Largo máximo caparazón (mm) } & \multicolumn{4}{c|}{ Peso seco de las hembras (g) } \\
Estadío & Promedio & $\pm \mathbf{D E}$ & Mínimo & Máximo & $\mathbf{n}$ & Promedio & $\pm \mathbf{D E}$ & Mínimo & Máximo & $\mathbf{n}$ \\
\hline I & 6,7 & 0,68 & 5,0 & 7,9 & 28 & 0,1207 & 0,044 & 0,04 & 0,21 & 28 \\
II & 6,8 & 0,61 & 4,4 & 8,0 & 15 & 0,1286 & 0,044 & 0,04 & 0,21 & 15 \\
III & 6,3 & 0,82 & 4,3 & 7,7 & 36 & 0,1229 & 0,043 & 0,06 & 0,20 & 36 \\
\hline
\end{tabular}

$\mathrm{DE}=$ desviación estándar

titud han sido descritos para otras especies del género Petrolisthes (Lardies y Wehrtmann, 1996) y para otras especies de decápodos (Clarke, 1979, 1982; Clarke et al., 1991; Gorny et al., 1992) . El rango en el número de huevos por hembra hallados en el presente trabajo (55-315 huevos) fue menor al mencionado por Sanhueza et al. (1975) aunque las tallas de hembras ovígeras fueron similares. Sin embargo dado que en el presente trabajo se realizó el muestreo en marzo solamente, es probable que, el valor más alto obtenido no representa el máximo posible para esta población, pero sí se puede afirmar que el valor más bajo se encuentra por debajo del mínimo obtenido en El Tabo, que fue de 75 huevos (Sanhueza et al., 1975).

El rango de portación de huevos determinado para $P$. granulosus es similar al observado en otros Porcelánidos chilenos (Lardies y Wehrtmann, 1996) y en $P$. armatus, $P$. haigae y $P$. zacae (Reid y Corey, 1991), aún cuando estas especies son de distribución tropical y subtropical.

Por otra parte, el número de huevos por hembra está estrechamente relacionado con la talla del ejemplar, aspecto que se observa en $P$. laevigatus (Lardies y
Tabla 2. Valores determinados de número de huevos, volumen por huevo, volumen de la masa de huevos y RO (esfuerzo reproductivo) en relación a las tres categorías de desarrollo embrionario utilizadas.

Table 2. Number of eggs, volume per egg, egg mass volume and RO (reproductive output) values for the three embrionic development stages used.

\begin{tabular}{|lccc|}
\hline \multicolumn{1}{|c}{ Estadío I } & Estadío II & Estadío III \\
\hline Número de huevos & & & \\
Promedio & 179,14 & 187,00 & 168,86 \\
\pm DE & 73,60 & 53,38 & 63,25 \\
Mínimo & 65 & 55 & 72 \\
Máximo & 315 & 277 & 300 \\
n & 28 & 15 & 36 \\
Volumen por huevo $\left(\mathbf{m m}^{3}\right)$ & & & \\
Promedio & 0,096 & 0,104 & 0,140 \\
\pm DE & 0,021 & 0,019 & 0,026 \\
Mínimo & 0,062 & 0,08 & 0,09 \\
Máximo & 0,14 & 0,15 & 0,24 \\
$\mathrm{n}$ & 28 & 15 & 36 \\
Volumen masa de huevos & & & \\
Promedio & 17,46 & 19,51 & 23,83 \\
\pm DE & 8,59 & 6,73 & 11,00 \\
Mínimo & 4,99 & 6,27 & 8,32 \\
Máximo & 37,95 & 31,20 & 65,68 \\
$\mathrm{n}$ & 28 & 15 & 36 \\
RO (total = 0,1033 $\pm \mathbf{0 , 0 6 )}$ & & & \\
Promedio & 0,1074 & 0,1278 & 0,0891 \\
DE & 0,0607 & 0,0644 & 0,055 \\
$\mathrm{n}$ & 28 & 15 & 36 \\
\hline DE & & & \\
\hline
\end{tabular}

$\mathrm{DE}=$ desviación estándar 
Tabla 3. Relación porcentual relativa entre el grado de desarrollo embrionario (estadíos I, II y III) y la coloración de la masa de huevos. M: huevos de color morado, MR: huevos de color morado-rojizo, MA: huevos de color morado anaranjado, n: número de hembras cuyos huevos correspondieron a cada estadío embrionario.

Table 3. Relative percent relationship between the embryonic degree of development (stages I, II and III) and egg-mass coloration. M: pink eggs, MR: pink-reddish eggs. MA: pink-orange eggs, n: number of females per each embryonic stage.

\begin{tabular}{|ccccc|}
\hline \multicolumn{5}{c|}{ Coloración de la masa de huevos } \\
Estadío & Morado (\%) & Morado rojizo (\%) & Morado anaranjado (\%) & n \\
\hline I & 96,42 & 3,57 & 0 & 28 \\
II & 33,33 & 67,67 & 0 & 15 \\
III & 0 & 13,89 & 86,11 & 36 \\
\hline
\end{tabular}

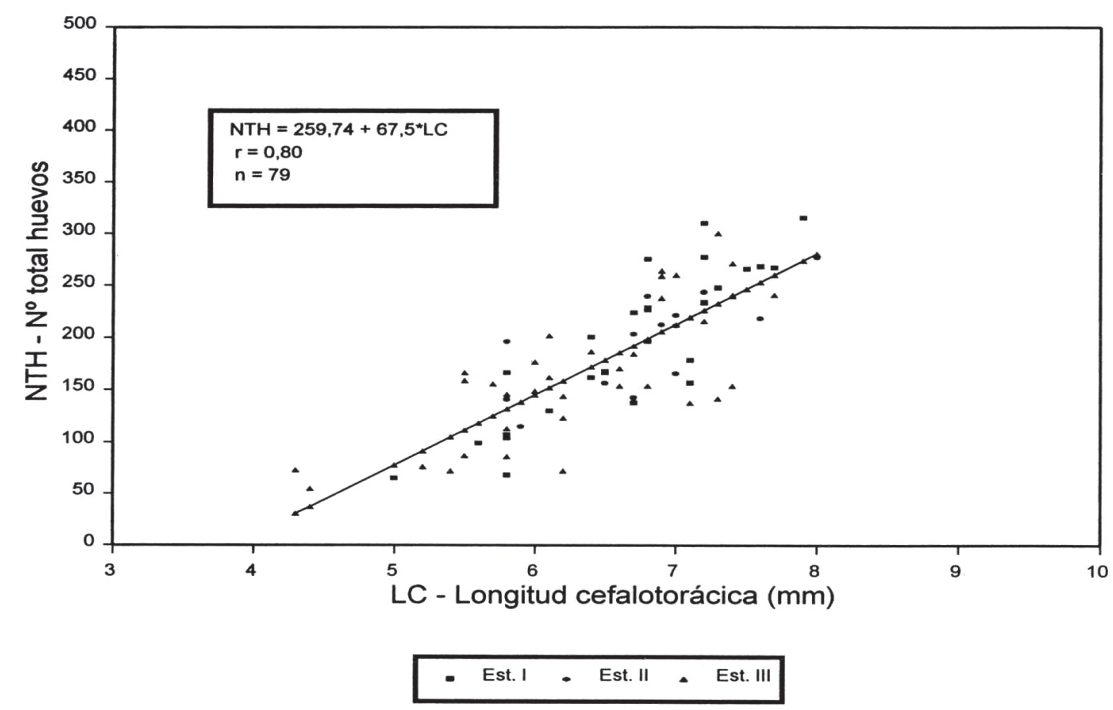

Figura 1. Fecundidad realizada o número total de huevos en función de la longitud cefalotóracica, por estadío de desarrollo embrionario, en Petrolisthes granulosus.

Figure 1. Actual fecundity or total number of eggs as a function of the cephalotoraxic length, by development stage, of Petrolisthes granulosus.

Wehrtmann, 1996) y en otros grupos de crustáceos (Attard y Hudon, 1987; Hines, 1982, 1988, 1991, 1992).

Al estudiar la media del número de huevos por estadío se encontró que no existe una pérdida significativa conforme progresa el desarrollo embrionario, a diferencia de lo reportado para $P$. laevigatus con una pérdida de $13,8 \%$ (Lardies y Wehrtmann, 1996); P. violaceus con $17,7 \%$
(Quiroga, com. pers.) y de un $15 \%$ en P. elongatus (Kuris, 1991).

Pese a la diferencia no significativa encontrada entre el estadío I y el II, el aumento del número de huevos entre estos estadíos podría deberse a que las hembras ovígeras del estadío I no han completado aún el desove, por lo que la cantidad de huevos encontrados correspondería a una subestimación del valor real; esto podría contrastarse histológicamente 
mediante el estudio de la maduración oocitaria. Por otra parte, podría suceder que no existiera pérdida de huevos durante el desarrollo embrionario o que el número de ejemplares procesados no fuera lo suficientemente grande como para detectar diferencias estadísticamente significativas.

A partir de los resultados obtenidos para el volumen medio de los huevos por estadío, se puede concluir que existe un aumento significativo entre los estadíos I y III. El aumento total registrado fue del 45,8\%, valor semejante al obtenido en $P$. laevigatus con un incremento de 40,5\% (Lardies y Wehrtmann, 1996), y en P. violaceus de un $37,8 \%$ (Quiroga, com. pers.). También es importante destacar que entre los estadíos I y II no se encontraron diferencias significativas, y aunque esto podría reflejar un hecho real debería considerarse la posibilidad de combinar caracteres morfológicos y morfométricos de los huevos que aumentando el número de estadíos descritos podrá minimizar la subjetividad del criterio utilizado.

El RO (peso seco) presentó un valor del 0,1033, similar al citado por Hines (1982, 1989, 1992) para veinte especies de familias de braquiuros. El obtenido en el presente trabajo es ligeramente mayor si se le compara con otras especies del género Petrolisthes, en las que el valor del RO fue de 0,049 en $P$. violaceus (Quiroga, com pers.) y en 0,057 en P. laevigatus (Lardies y Wehrtmann, 1996). Si se compara el RO por estadío embrionario, se encuentra una disminución del 24,3\% hacia el último estadío, que está en relación con el decremento en el peso seco de la masa ovígera, dado que los pesos medios por estadío (de la hembra) son constantes.

La disminución en el contenido de materia orgánica ha sido observada para P. laevigatus (Lardies y Wehrtmann, 1996), P. violaceous (Quiroga, com. pers.) y Chasmagnathus granulata (Stella et al., 1996), entre otros. Esta disminución hacia fines del desarrollo embrionario estaría relacionada con el consumo de vitelo para su transformación en estructuras embrionarias (Dworschak, 1988), como así también por un mayor metabolismo larval.

Sin embargo, aún existiendo una disminución en el peso seco de la masa ovígera, el incremento del volumen del huevo hacia el estadío III es significativamente mayor, lo que indica que este aumento se debe a un aumento en el contenido de agua. Éste podría estar vinculado a dos procesos no necesariamente antagónicos. Por un lado, la formación de tejidos a partir del catabolismo del vitelo podría estar incrementando la cantidad de metabolitos libres y requerir mayor cantidad de agua para su solvatación (Stella et al., 1996); por otra parte el ingreso de agua hacia fines del desarrollo embrionario se podría relacionar con el incremento de la presión osmótica interna, mecanismo independiente ó simultáneo con los movimientos del embrión que favoreciera la eclosión larval (Lardies y Wehrtmann, 1996; Valdés et al., 1991). El “mecanismo osmótico" para la eclosión larval ha sido también descrito en Artemia (Green, 1965), copépodos (Davies, 1959) y crustáceos en general, al menos como un mecanismo parcial (Kidd, 1991).

Como consecuencia de lo expuesto anteriormente, sería de esperar que si el volumen por huevo aumenta y el peso de la masa ovígera seca disminuye de los estadíos I-II al III, el volumen medio de la masa ovígera total presentará un incremento hacia el estadío III. En P. armatus la masa ovígera alcanza un incremento de hasta $66,9 \%$ entre los estadíos temprano y tardío de desarrollo embrionario (Reid y Corey, 1991); en P. laevigatus el incremento fue de $56,6 \%$.

En el presente caso, aunque las diferencias entre el volumen medio de la masa ovígera total entre estadíos no fueron estadísticamente significativas, la diferencia observada entre el estadío final e inicial fue del 36,5\%. Dado que el tcalc $=-2,3$ es muy próximo al valor crítico, sería conveniente incrementar el número de ejemplares analizados para confirmar los resultados obtenidos o elegir un test de mayor sensibilidad.

\section{AGRADECIMIENTOS}

A los profesores Raúl Soto, Ingo Wehrtmann, Gabriel Claramunt y Rosalino Fuenzalida y a todos los integrantes de los laboratorios, biblioteca, secretaría y auxiliares de la Universidad Arturo Prat que colaboraron con este trabajo y que hicieron más grata nuestra estadía en esa institución. A la Fundación Andes por su apoyo económico para la realización del Curso de Postgrado organizado por la Universidad Austral de Chile junto con la Universidad Arturo Prat y a la DAAD por el subsidio brindado a Teresa Jerí. 


\section{REFERENCIAS}

Annala, J. 1991. Factors influencing fecundity and population egg production of Jasus species. In: A. Wehner and A. Kuris (eds.), Crustacean Egg Production. Crustacean Issues 7, F.R. Schram, A. Balkema, Rotterdam/Brookfield, pp. 301-315.

Antezana, T., E. Fagetti y M.T. López. 1965. Observaciones biológicas en decápodos comunes de Valparaíso. Rev. Biol. Mar., 12 (1-3): 1-60.

Attard, J. y C. Hudón. 1987. Embryonic development and energetic investment in egg production in relation to size of female lobster (Homarus americanus). Can. J. Fish. Aquat. Sci., 44: 11571164.

Clarke, A. 1979. On living in cold water: K-strategies in Antarctic benthos. Mar. Biol., 55: 111-119.

Clarke, A. 1982. Lipid synthesis and reproduction in the polar shrimp Chorismus antarcticus. Mar. Ecol. Prog. Series, 9: 81-92.

Clarke, A., C. Hopkins y E. Nilssen. 1991. Egg size and reproductive output in the deep-water prawn Pandalus borealis Kryer, 1838. Funct. Ecol., 5: 724-730.

Corey, S. y D. Reid. 1991. Comparative fecundity of decapod crustaceans. I. The fecundity of thirtythree species of nine families of caridean shrimps. Crustaceana, 60(3): 270-294.

Davies, C. 1959. Osmotic hatching in the eggs of some fresh-water copepods. Biol. Bull., 116: 15-29.

Dworschak, P. 1988. The biology of Upogebia pusilla (Petagna) (Decapoda, Thalassinidea). III. Growth and production. Mar. Ecol., 9: 51-77.

Gorny, M., W. Arntz, A. Clarke y D. Gore. 1992. Reproductive biology of caridean decapods from the Weddell Sea. Polar Biol., 12: 111-120.

Green, J. 1965. Chemical embryology of the Crustacea. Biol. Rev., 40: 580-600.

Haig, J. 1960. The Porcellanidae (Crustacea, Anomura) of the southeastern Pacific. Allan Hancock Pac. Exp., 24: 1-440.

Hines, A. 1982. Allometric constraint and variables of reproductive effort in brachyuran crabs. Mar. Biol., 69: 309-320.
Hines, A. 1988. Fecundity and reproductive output in two species of deep-sea crabs, Geryon fenneri and G. quinquedens (Decapoda: Brachyura). J. Crust. Biol., 8 (4): 557-562.

Hines, A. 1991. Fecundity and reproductive output in nine species of Cancer crabs (Crustacea, Brachyura, Cangridae). Can. J. Fish. Aquat. Sci., 48: 267-275.

Hines, A. 1992. Constraint on reproductive output in brachyuran crabs: pinnotherids test the rule. Amer. Zool., 32: 503-511.

Ivanova, M. y S. Vassilenko. 1987. Relationship between number of eggs, brood weight, and female body weight in Crustacea. Int. Revue. ges. Hydrobiol., 72: 147-169.

Kidd, R. 1991. Development of embryonic and naupliar setae and spines and their role in hatching in the penaeid Sicyonia ingentis: a light and electron microscopic study. J. Crust. Biol., 11: 40-55.

Kuris, A. 1991. A review of patterns and causes of crustacean brood mortality. In: A. Wenner and A. Kuris (eds.), Crustacean Egg Production, Crustacean Issues 7, F.R. Schram, A. Balkema, Rotterdam/ Brookfield, pp. 117-141.

Lardies, M. y I. Wehrtmann. 1996. Aspects of the reproductive biology of Petrolisthes laevigatus (Guérin, 1835) (Decapoda, Anomura, Porcellanidae). Part I: Reproductive output and chemical composition of eggs during embryonic development. Arch. Fish. Mar. Res., 43(2): 121-135.

Reid, D. y S. Corey. 1991. Comparative fecundity of decapod crustaceans II. The fecundity of fifteen species of Anomuran and Brachyuran crabs. Crustaceana. 61: 175-189.

Sanhueza, E., N. Bahamonde y M.T. López. 1975. Petrolisthes granulosus (Guérin) en biocenosis supramareales de El Tabo (Crustacea, Decapoda, Anomura). Bol. Mus. Nac. Hist. Nat., 34: 121-136.

Stechey, D. y K. Somers. 1995. Potential, realized, and actual fecundity in the crayfish Orconectes immunis from southwestern Ontario. Can. J. Zool., 73: 672677.

Stella, V., L. López y E. Rodríguez. 1996. Reproductive parameters in the estuarine crab Chasmagnathus granulata (Decapoda, Brachyura, Grapsidae). Crustaceana, 69(3): 306-312. 
Turner, R. y J. Lawrence 1979. Volume and composition of echinoderm eggs: implication for the use of egg size in life history models. In: W.E. Staneyk (ed.), Reproductive ecology of marine invertebrates. Contr. Belle W. "Baruch Libr. Mar. Sci. 9". Univ. South Caroline Press Columbia, pp. 25-40.

Valdés, L., M. Alvarez-Osorio y E. González-Gurriaran. 1991. Incubation of eggs of Necora puber (L., 1767) (Decapoda, Brachyura, Portunidae). Volume and biomass changes in embrionic development. Crustaceana, 60(2): 163-177.

Recibido el 13 de mayo de 1996.

Aceptado el 23 de diciembre de 1996.
Viviani, C. 1969. Los Porcellanidae (Crustacea: Anomura) chilenos. Beitz. z. Neotrop. Fauna, 6(1): 40-56.

Wehrtmann, I. 1990. Distribution and reproduction of Ambidexter panamense and Palaemonetes schmitii, in Pacific Costa Rica (Crustacea: Decapoda). Rev. Biol. Trop., 38(2A): 327-329. 